\title{
LA GESTIÓN DE RIESGOS DESDE LA PSICOLOGÍA SOCIAL
}

\author{
Ariel Osvaldo Quezada Len ${ }^{1}$
}

\section{Resumen}

El concepto de vulnerabilidad es de vital importancia para la psicología, dado que articula la relación entre aspectos del individuo y del ambiente físico y social, como elementos claves para el bienestar de la persona. La vulnerabilidad puede analizarse desde distintos puntos de vista complementarios, para tener una comprensión más cabal. La vulnerabilidad, en su interjuego entre la amenaza y la capacidad, conforman un todo relacionado que articula el concepto de riesgo, el que puede administrarse con la finalidad de reducir la probabilidad de deteriorar la calidad de vida de las personas. En este apartado se brindarán definiciones centrales, que ayudarán a configurar un apropiado mapa conceptual, que ayude al lector a sentar las bases elementales de una administración de riesgos. El foco está centrado en la seguridad industrial, dado que los orígenes de la disciplina están ligados a este campo, para después decantar en su evaluación estandarizada y el monitoreo continuo de los riesgos psicosociales, con una creciente importancia, acorde con la evolución de las características del trabajo. Finalmente, se abordará el concepto de resiliencia, el cual, inicialmente descrito como una capacidad individual, se ha extendido a grupos y organizaciones. La resiliencia se erige como complemento a la vulnerabilidad, dado que proporciona un balance en favor de la protección y salud mental de las personas.

Palabras clave: riesgo, amenaza, vulnerabilidad, capacidad, resiliencia

1 Jefe de Carrera de Psicología / Escuela de Ciencias Jurídicas y Sociales - Universidad Viña del Mar - Chile. MIND Servicios de Psicología y Consultorías Limitada, Chile. Avenida Coraceros 50, Viña del Mar, CP 2520000, +56993300419, aquezada@mindchile.cl, Licenciado en Psicología (Universidad de Valparaíso), Doctor en Psicología Social (Universitat de Barcelona), Magister en Gestión de Organizaciones (Universidad Adolfo Ibáñez). Psicología Social, Influencia Social, Seguridad en Organizaciones, Riesgos Psicosociales Laborales, Control de Fatalidad 


\section{Abstract}

The concept of vulnerability is of vital importance for psychology, since it articulates the relationship between aspects of the individual and the physical and social environment, as key elements for the wellbeing of the person. The vulnerability can be analyzed from different complementary points of view, to have a more complete understanding. Vulnerability, in its interplay between threat and capacity, make up a related whole that articulates the concept of risk, which can be managed in order to reduce the likelihood of deteriorating the quality of life of people. In this section, central definitions will be provided, which will help to configure an appropriate conceptual map that will help the reader lay the basic foundations of risk management. The focus is on industrial safety, given that the origins of the discipline are linked to this field, to later decant in its standardized evaluation and continuous monitoring of psychosocial risks, with an increasing importance, according to the evolution of the characteristics from work. Finally, the concept of resilience will be addressed, which, initially described as an individual capacity, has been extended to groups and organizations. Resilience is a complement to vulnerability, given that it provides a balance in favor of the protection and mental health of people.

Keywords: risk, threat, vulnerability, capacity, resilience

\section{Hacia una conceptualización inicial}

Los seres humanos somos una expresión última de una serie de ajustes adaptativos a nuestro entorno. En efecto, capacidades físicas y psicológicas son expresión de esta evolución que hemos tenido como especie. Un ejemplo de esta evolución es la capacidad de aprendizaje, que ha permitido la adaptabilidad y supervivencia de la especie a través de los años y enfrentando distintos contextos (Domjan, 2010).

En esta perspectiva, la ponderación de los beneficios y los riesgos de una acción podría mantener una relación con nuestra evolución dado que, en condiciones normales, se privilegiará la realización de conductas que proporcionen beneficios sin pagar un costo muy alto o que atenten contra la integridad del individuo.

Para tener una mayor precisión de los términos que se emplearán, se hace necesario aclarar algunos conceptos asociados en esta problemática. 
Para comenzar, el evento activador se podría denominar desastre, concepto anclado en un particular origen etimológico griego: "des" "aster". Dado que las comunidades eran asiduas a la observación de los astros y distintos fenómenos de los cuerpos celestes, se creía que tenían una relación directa con diversos fenómenos en la tierra y en la vida de las personas. Así, la negación de los astros (en este caso, un desastre) estaría ligado al augurio de calamidades y fatalidades para la comunidad. Más tarde, al surgir las religiones, estos desastres se asociaron a la idea de un castigo de las divinidades por alejarse del camino dictaminado o, en caso contrario, la no ocurrencia de un desastre se debe precisamente a la intervención protectora de la divinidad (Ulloa, 2011).

Desde el punto de vista de las personas un desastre corresponde a una alteración de la vida cotidiana y que no puede restaurarse por los propios medios, requiriendo ayuda externa. Esta alteración puede ser generada por el impacto de un fenómeno de la naturaleza o de la acción humana, que incide directamente en el funcionamiento de una sociedad (Ulloa, 2011).

El que se desencadene un desastre está también asociado dinámicamente a otros tres conceptos centrales: amenaza, vulnerabilidad y capacidad.

Amenaza (o peligro) corresponde a fenómenos extremos, de origen natural o no, que actúan en un determinado espacio y tiempo definido, con el potencial de causar daño a alguien. De manera simple, son fenómenos que tienen el potencial de afectar negativamente a los seres humanos.

Vulnerabilidad, por su parte, se identifica con la propensión de un elemento expuesto, a sufrir daños (D’Ercole \& Trujillo, 2003). Es decir, que una amenaza genere daño está íntimamente relacionado con que la susceptibilidad de una persona o un grupo se vean afectados. En términos formales, la vulnerabilidad son las características y las circunstancias de una persona, comunidad o sistema que los hacen susceptibles a los efectos perjudiciales de una amenaza.

Las capacidades son la sumatoria de todas las fortalezas, atributos y recursos disponibles dentro de una persona, comunidad, sociedad u organización que pueden emplearse para mantener la vida cotidiana y enfrentar las amenazas con sus potenciales consecuencias negativas. La amplia gama de las capacidades va desde la infraestructura, los medios 
físicos, las instituciones, las habilidades de afrontamiento conocimiento, capital y destrezas individuales y comunitarias (UNESCO, 2012).

Teniendo claros los conceptos ya definidos, el riesgo podría expresarse como una función tal como lo muestra la Figura 1:

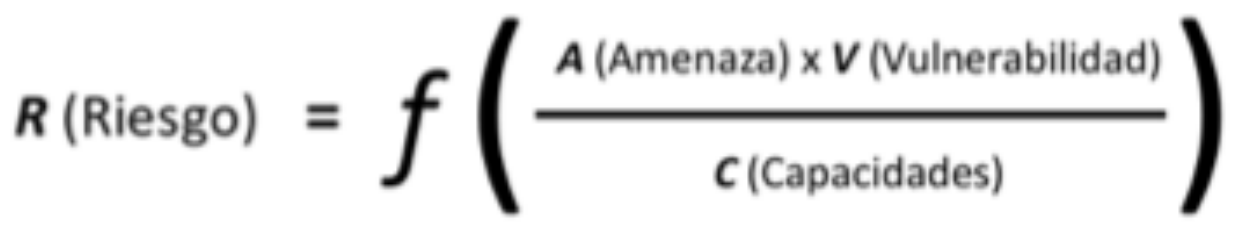

Figura 1. Conformación del riesgo en función de la relación entre la presencia de amenazas, vulnerabilidad y capacidades.

A modo de ejemplo cotidiano, podría ilustrarse en el riesgo (R) de sufrir una insolación se daría en la interacción entre una amenaza (A) de alta radiación solar x una vulnerabilidad (V) de estar al exterior y tener una piel blanca. Ese riesgo (R) podría aumentar o disminuir en función de las capacidades del individuo (C) ligadas a la posibilidad de cubrirse del sol y/o aplicar algún filtro solar sobre la piel.

Ejemplificándolo en el caso de un riesgo psicosocial de tipo laboral, estaría asociado a una amenaza ligada a condiciones laborales de alta exigencia, vulnerabilidades individuales tales como la baja experiencia laboral y capacidades del trabajador relacionadas, por ejemplo, a la capacidad de aprendizaje y las habilidades sociales.

Como se puede apreciar, el concepto de riesgo no es absoluto ni inmutable. Al contrario, es un concepto dinámico y que se relaciona con las amenazas, vulnerabilidades y capacidades del sujeto, grupo o comunidad que se desea analizar.

\section{Gestión de riesgos y la cultura de seguridad}

Si se considera que la magnitud de un riesgo podría aumentar o disminuir dependiendo de cómo se enfrentan las amenazas, se minimizan las vulnerabilidades y se fortalecen las capacidades, se puede establecer una apropiada gestión de riesgos. Es más, los riesgos se pueden evaluar, medir y controlar con distintos grados de efectividad. 
El psicólogo británico James Reason es seguramente uno de los más citados en relación con la seguridad y cómo se genera el error humano que, finalmente, se traduce en un desastre o incidente. Uno de los modelos largamente difundidos es el del Queso Suizo, en el cual se observa que un incidente es un evento que logró traspasar cada una de las medidas y acciones que una organización o comunidad estableció para evitar un accidente. En la Figura 2 se observa gráficamente cómo el evento indeseable ha sorteado las medidas de control que se definieron para evitarlo.

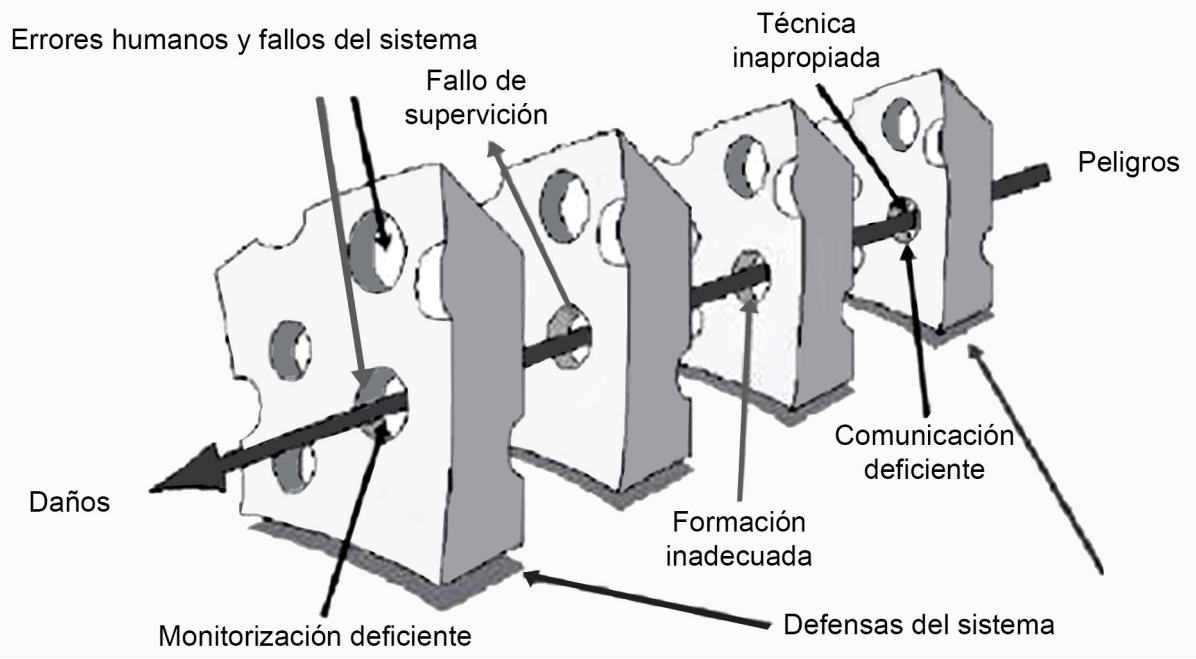

Figura 2. El Modelo del Queso Suizo es una representación gráfica de cómo un evento indeseable que concluye en un accidente logró sortear los controles definidos por el sistema. Extraído de Ministerio de Protección Social (2007)

Tal como se puede apreciar, el daño ocurrió toda vez que un peligro traspasó las barreras de una ejecución y procedimientos técnicos, sistemas de comunicación, inadecuada formación o instrucción, fallas en la supervisión y en la monitorización.

Siguiendo con esta distinción, existen tipos de acciones humanas que pueden decantar en accidentes y que, para mitigar su ocurrencia, el sistema debería brindar las barreras suficientes para limitar su impacto (Reason, Manstead, Stradling, Baxter, \& Campbell, 1990). Esquemáticamente se puede observar con mayor claridad en la Figura 3. 


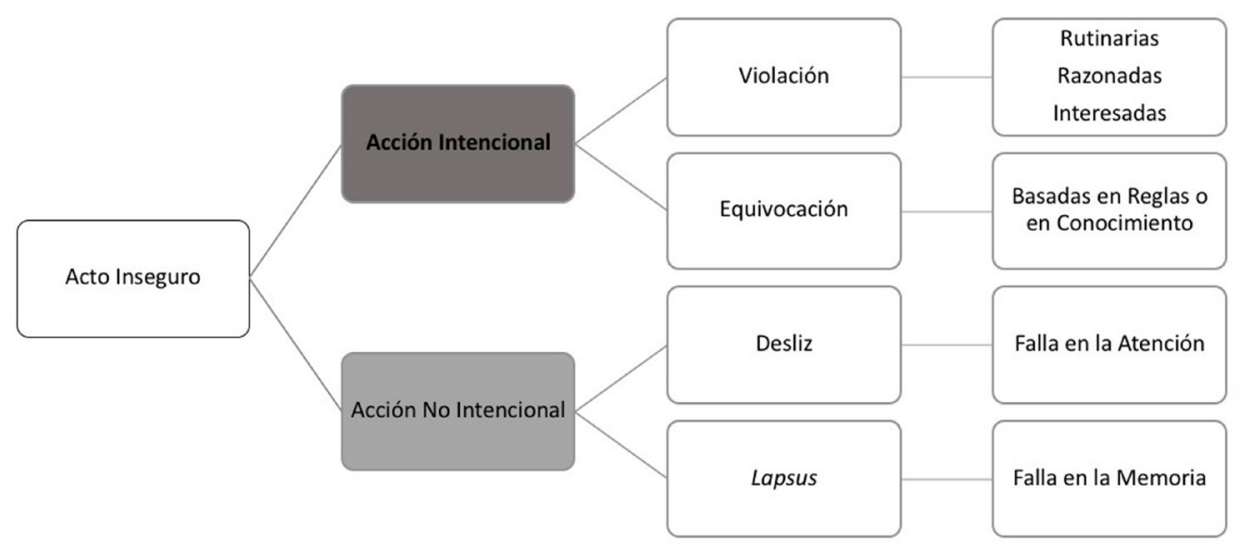

Figura 3. Taxonomía en que se distingue los tipos de acciones que pueden estar a la base de accidentes según Reason et al. (1991).

La primera distinción que abre el árbol taxonómico se focaliza en la intencionalidad de la acción, es decir, si es o no una conducta deliberada por parte de la persona. Si es deliberada, esta puede corresponder a una equivocación, en la cual hay una clara voluntad de realizar una conducta, pero que se basa en reglas o en conocimientos errados, a diferencia de la violación, que es una acción destinada a romper una regla o un procedimiento. En la otra banda se encuentran las acciones no intencionales, en los que el sujeto no tiene la voluntad de realizar un acto inseguro pero que ocurre a causa de una falla de memoria —en el caso del lapsus- o en una falla en la atención -en el caso de un Desliz- (Reason et al., 1991). Ejemplificando cada uno de estas acciones humanas en el ámbito de la conducción, una violación sería entrar en contrasentido de una calle para hacer una ruta más corta, teniendo completo conocimiento de que el sentido es el opuesto. Equivocación se podría dar con la misma conducta de ingresar en contrasentido, pero, por desconocer que el sentido de la calle es opuesto, caso que se da en rutas internas en faenas industriales o, de manera más dramática, cuando un conductor de Europa Continental ingresa a rutas británicas pensando que el sentido de la conducción es la misma. Una acción no intencional del tipo desliz puede darse cuando, al conducir alguien, toma un vaso de café que se derrama perdiendo el control de su vehículo. Lapsus podría ejemplificarse al realizar una ruta y haber olvidado el camino correcto para llegar a destino. Esta taxonomía es de gran ayuda para poder dar un efectivo control de la ocurrencia de actos 
inseguros, con estrategias diferenciales, dependiendo intencionalidad de ellos.

Para evitar que acciones humanas o condiciones del entorno se traduzcan en un desastre, surge la Gestión de Riesgos. Se pueden distinguir tres grandes tipos de Gestión (Ulloa, 2011):

Gestión Reactiva: Cuando se presenta una emergencia, se responde contingentemente a la emergencia, asumiendo los costos y tratando de minimizar los daños.

Gestión Correctiva: Se adoptan medidas y acciones con anticipación, para lograr conseguir una reducción en las condiciones de riesgos ya existentes. Se aprovechan los análisis de riesgos previos, dando cuenta de una memoria histórica ante desastres previos, con el fin de revertir y cambiar los procesos que se asocian a los riesgos.

Gestión Prospectiva: Consiste en la adopción de medidas y acciones que lleven a una planificación del desarrollo para evitar la generación de nuevas condiciones de riesgo. Incluso, se aspira a que se desarrollen estrategias para riesgos que aún no existen, a través de regulaciones legales, inversiones públicas y privadas, formación y desarrollo de capacidades, etc.

Sin duda alguna, la Cultura de Seguridad de una organización o una comunidad obedece también a estadios de desarrollo y madurez característicos. Una buena evaluación permite conocer en qué etapa de madurez se encuentra la entidad, para poder ajustar las expectativas de lo que puede o no hacer y, en un futuro, preparar el desarrollo a estadios superiores.

Parker, Lawrie y Hudson, en 2006, desarrollan una útil caracterización del estado de madurez de la cultura de seguridad en organizaciones, la que también puede ser útil para la evaluación de estrategias nacionales y, aún más, en términos del autoanálisis individual. En la Figura 4 se puede apreciar con más detalle. 


\section{Escalera de la Cultura Preventiva}

La madurez de una organización depende su ubicación en la Escalera de la Cultura Preventiva

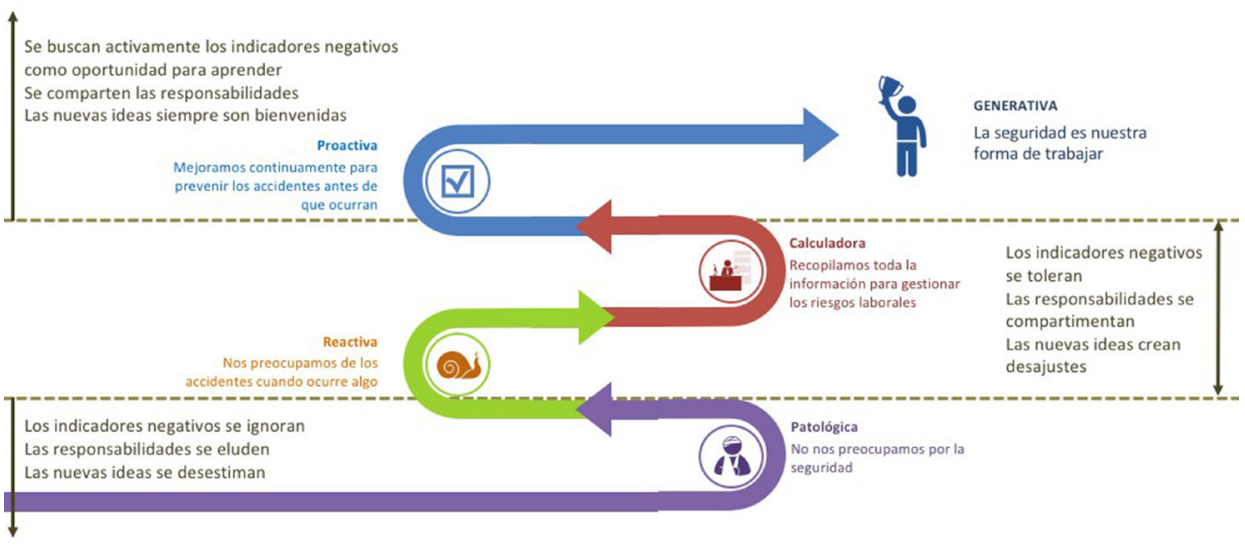

Figura 4. Cuadro en el que se visualizan los cinco estadios de maduración en Cultura de Seguridad de una organización, según Parker, Lawrie y Hudson (2006).

Como se observa en la Figura 4, en la madurez de una organización se pueden distinguir Organizaciones de Cultura Patológica, en la cual no hay una preocupación por la seguridad, en donde los indicadores negativos son ignorados, eludiendo cualquier responsabilidad y siendo refractarios a cualquier nueva idea tendiente a la mejora en la seguridad. Un escalón más arriba están aquellas Organizaciones de Cultura Reactiva, en las que se toman acciones siempre después de que haya ocurrido un evento indeseable. Más arriba, están las Organizaciones de Cultura Calculadora, en las que se toma registro de los incidentes, como parte de la gestión a las cuales están legalmente obligadas. En los estadios superiores se encuentra la Cultura Proactiva, en la que la mejora constante es la tónica para evitar que los accidentes ocurran. Finalmente, la Cultura Generativa es aquella en la que la seguridad es el valor que cruza la forma de actuar de las personas (Parker et al., 2006).

La gestión de riesgos es una ciencia aplicada, que ha surgido principalmente al alero de la industria en donde destacados psicólogos han hecho un importante aporte teórico y práctico. Sin embargo, este desarrollo puede extrapolarse a distintas áreas aplicadas en las que la meta principal es reducir la ocurrencia de desastres que lesionan la calidad de vida de las personas. En este sentido, siempre que sea posible identificar amenazas, 
evaluar las vulnerabilidades y mejorar la capacidad de respuesta, se estará haciendo una gestión de riesgos.

A continuación, se revisará cómo se identifican y se manejan los riesgos psicosociales en el ámbito laboral.

\section{Riesgos psicosociales laborales}

El mundo del trabajo es largamente el ambiente en donde se destina la mayor cantidad de horas de vida y se erige como uno de los más relevantes en la vida de la persona.

La seguridad en el trabajo dedica grandes esfuerzos en la eliminación de la fatalidad en el trabajo como también la reducción de las enfermedades profesionales ocasionadas en el trabajo. Esta es la razón por la cual los países han diseñado protocolos vigilancia de salud ocupacional para el control de enfermedades. A modo de ejemplo, en el caso de particular de la minería en Chile (Instituto de Seguridad Laboral, 2017), los obligatorios son los de pérdida auditiva por exposición a ruido en el trabajo (PREXOR), los de enfermedades por exposición a sílice (PLANESI), el de identificación y evaluación de factores de riesgo de trastornos músculo-esqueléticos relacionados con el trabajo (TEMERT) y, finalmente, el Protocolo de Riesgos Psicosociales en el Trabajo (RPSL).

En menos de medio siglo el trabajo ha tenido una notable evolución de la mano de grandes cambios económicos, políticos, tecnológicos y sociales. En efecto, el trabajo de mayor demanda mental ha ido ganando mayor proporción, siendo más quienes trabajan en oficinas que quienes lo hacen en industrias o en el campo (Superintendencia de Seguridad Social, 2016). En esta lógica, se ha hecho progresivamente más importante desarrollar habilidades de tipo interpersonales (comunicación) y cognitivas (por ejemplo, toma de decisiones). La estabilidad en el empleo se ha reducido, valorando la flexibilidad del trabajador y demandando de este que pueda afrontar cambios, sea proactivo y comprometido, a veces a costa de la vida personal y familiar. Sin que hayan desaparecido los riesgos de tipo físico, químico, biológico o ergonómico, la importancia de los Riesgos Psicosociales Laborales cobra una creciente importancia.

La Organización Internacional del Trabajo, en 1984, describe un modelo de factores psicosociales en el trabajo, en el que la interacción entre 
las características de la organización y las características del grupo humano podría tener consecuencias favorables o desfavorables en la satisfacción laboral, el rendimiento y, fundamentalmente, en la salud de los trabajadores. Este modelo se ilustra en la Figura 5.

\section{Factores Psicosociales}

Oficina Internacional del Trabajo

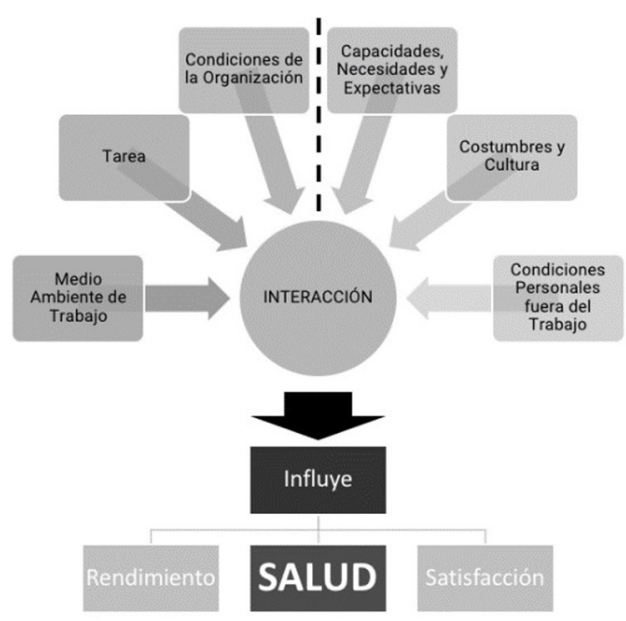

Figura 5. Modelo de Factores Psicosociales en el Trabajo, elaborado por la OIT (1984).

Identificado un modelo apropiado para entender la incidencia en la Salud Mental de los trabajadores, el paso siguiente es poder establecer un instrumento de medición que permita evaluar el riesgo y, en consecuencia, poder mantenerlo controlado. Ante este desafío, surgen una serie de esfuerzos para poder medir el riesgo de contraer un trastorno de salud mental en el trabajo.

Una de las herramientas de medición más aceptadas es la Copenhagen Psychosocial Questionnaire (COPSOQ), luego adaptada al español en el Instituto Sindical de Trabajo, Ambiente y Salud de Barcelona (ISTAS). Finalmente, para la evaluación en Chile, la Superintendencia de Seguridad Social desarrolla el Cuestionario SUSESO/ISTAS 21 en sus versiones breve y completa (Superintendencia de Seguridad Social, 2016).

Las cinco dimensiones que se evalúan son las que se describen en la Tabla 1. 
Tabla 1

Dimensiones Evaluadas en el Cuestionario SUSESO/ISTAS 21 Breve

\begin{tabular}{|c|c|l|}
\hline Nombre de la Dimensión & Ítems & Descripción de la Dimensión \\
\hline Exigencias Psicológicas & 5 & $\begin{array}{l}\text { Exigencias Cuantitativas, } \\
\text { Cognitivas, Sensoriales, } \\
\text { Emocionales y Supresión de } \\
\text { Emociones }\end{array}$ \\
\hline $\begin{array}{c}\text { Trabajo Activo y Posibilidades de } \\
\text { Desarrollo }\end{array}$ & 5 & $\begin{array}{l}\text { Influencia, Control sobre el } \\
\text { tiempo de trabajo, Desarrollo en } \\
\text { el trabajo, Sentido del trabajo e } \\
\text { Integración en la empresa }\end{array}$ \\
\hline $\begin{array}{c}\text { Apoyo social en la empresa y } \\
\text { calidad de liderazgo }\end{array}$ & 5 & $\begin{array}{l}\text { Claridad de Rol, Conflicto de } \\
\text { Rol, Calidad de Liderazgo, } \\
\text { Calidad de la Relación con } \\
\text { Superiores y con compañeros de } \\
\text { trabajo. }\end{array}$ \\
\hline Compensaciones & 3 & $\begin{array}{l}\text { Estima, Inseguridad respecto } \\
\text { al contrato del trabajo y a las } \\
\text { características del trabajo }\end{array}$ \\
\hline Doble Presencia & 2 & $\begin{array}{l}\text { Preocupación por las Tareas } \\
\text { Domésticas }\end{array}$ \\
\hline
\end{tabular}

Esta evaluación se puede realizar en toda unidad de trabajo sobre 25 trabajadores. La aplicación es anónima y dependiendo del puntaje que, individualmente, un trabajador pueda obtener en cada una de las dimensiones, podría indicar el riesgo psicosocial que él percibe. Si más del 50\% de los trabajadores de una misma unidad muestran también un riesgo alto en esa dimensión, la unidad arroja una alerta y se consigna +1. En el caso opuesto, si más del 50\% de los trabajadores de una misma unidad indican un riesgo bajo, la dimensión arroja un riesgo bajo y se anota un -1. Un riesgo psicosocial alto, medio o bajo se determinará por la puntuación completa que tenga una unidad de trabajo en la suma de los +1 y -1 obtenidos en las cinco dimensiones. A partir de los resultados globales obtenidos se asocian acciones de mitigación determinadas. Esto se puede clarificar de mejor manera tal como aparece en la Figura 6. 


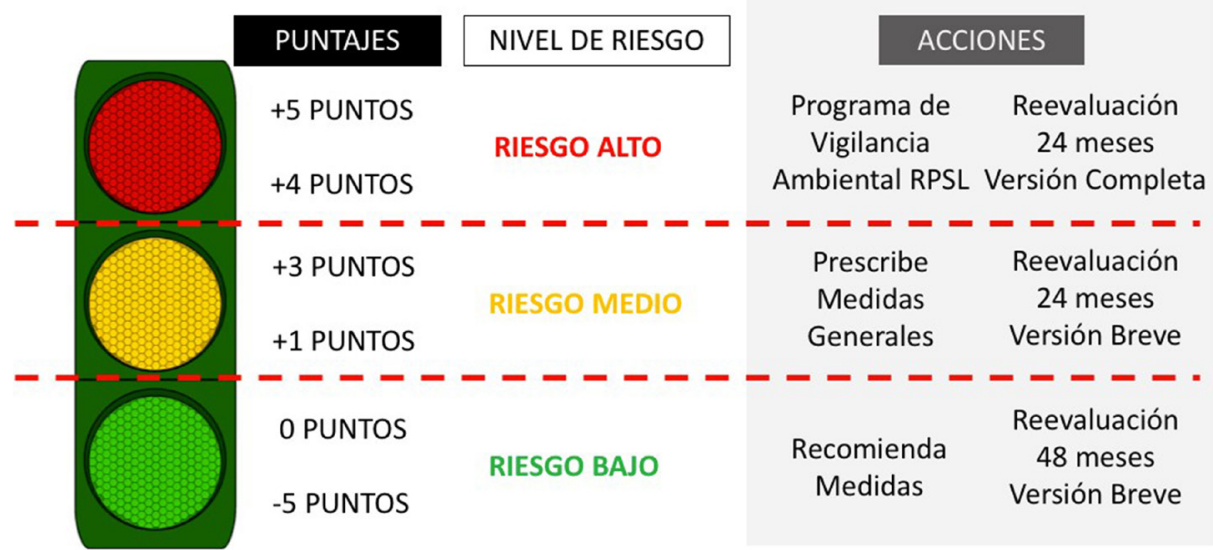

Figura 6. Relación entre los puntajes obtenidos en el Cuestionario SUSESO/ISTAS 21 versión breve, el nivel de riesgo y las acciones que la organización debe adoptar (Ministerio de Salud, 2017).

La aplicación de los protocolos de vigilancia laboral de riesgos psicosociales, brindará una valiosa información que permitirá caracterizar los riesgos más altos, las industrias y sus riesgos asociados, los trastornos de mayor frecuencia y los métodos de mitigación más eficientes.

\section{Resiliencia individual y de grupos y organizaciones}

El origen etimológico de la palabra resiliencia proviene del latín resilio y corresponde a la capacidad física de los objetos de rebotar o de absolver las perturbaciones para luego volver a la forma original (OMS, 1997). Sin embargo, una acepción más cercana a la psicología es aquella que describe a quienes, a pesar de nacer y desarrollarse en contextos de alto riesgo, se desarrollan psicológicamente sanos y exitosos.

Uno de los más destacados exponentes de la conceptualización y trabajo aplicado en el campo de la resiliencia, Michael Rutter (2012), señala que durante las dos últimas décadas la tendencia estuvo marcada por el cambio de foco desde el riesgo hacia la resiliencia por parte de investigadores, clínicos y actores de políticas públicas. En efecto, se privilegió enfatizar en aspectos positivos en lugar de hacerlos en aquellos desadaptativos, siguiendo una tendencia más amplia enmarcada en la Psicología Positiva. El mismo Rutter (2012) define la Resiliencia como la reducción de la 
vulnerabilidad a las experiencias de peligros ambientales, la superación de un estrés o la adversidad, o un resultado relativamente bueno a pesar de las experiencias de riesgo.

Se advierte entonces que la capacidad, enunciada anteriormente en la función de riesgo, tiene una directa relación con la resiliencia dado que proporciona una compensación a las vulnerabilidades que puede presentar una persona frente a una amenaza o peligro.

Si en los orígenes de esta conceptualización se focalizó en capacidades individuales de sujetos para enfrentar adversidades, recientemente se ha acuñado el término de Resiliencia Organizacional para describir la habilidad de una organización para recuperarse rápidamente, después de incidentes críticos o cambios ambientales repentinos, siendo incluso considerado directa o indirectamente como una medida de robustez organizacional (Xenidis \& Theocharous, 2014). Incluso, otras derivaciones han extendido su acepción para ligarla a la capacidad de reacción y de recuperación de una organización a diferentes cambios en el entorno, no solo físico, sino de negocios, alejándose completamente a su original vinculación a la salud (Kamlot, 2017).

En Iberoamérica, se han desarrollado interesantes equipos de investigación centrados en la promoción de organizaciones positivas que se comprometan activamente en la promoción de la salud con un enfoque comprehensivo, interdisciplinar y multicausal. Entre estos equipos se encuentra el de la Univesitat Jaume I en Castellón, España. Proponen que una organización positiva es aquella que incesantemente busca la excelencia organizacional y el éxito financiero, pero que van más allá dado que gozan de una fuerza laboral física y psicológicamente saludable capaz de mantener un ambiente laboral y cultura organizacional positivos. No solo pueden sortear períodos de cambio y turbulencia, sino que también tienen una alta capacidad de aprendizaje. En este equipo surge el concepto de Organización Saludable y Resistente o su sigla en inglés HERO por HEalthly $\mathcal{E}$ Resilient Organization (Salanova, Llorens, E Martínez, 2016). En esta línea, las Organizaciones Positivas son aquellas caracterizadas por el binomio: salud y resiliencia. Una organización de características HERO es aquella que realiza acciones sistemáticas, planificadas y proactivas, para mejorar tanto procesos como resultados de los trabajadores y la organización toda. Adicionalmente, son organizaciones resilientes, dado que mantienen un 
ajuste positivo frente a desafíos y se fortalecen ante situaciones adversas (Salanova et al., 2016).

Siguiendo en esta línea, el concepto de resiliencia no solo tiene aplicabilidad para la psicología individual, sino que también tiene perfecta sintonía con el mundo de las organizaciones, atendiendo a la creciente necesidad de contar con organizaciones tremendamente exitosas en términos empresariales, logro que se consigue cuando de base hay trabajadores con riesgos de salud mental acotados y se desarrollan en un ambiente laboral sano.

\section{Conclusión}

En este apartado se ha entregado una conceptualización que permite comprender la gestión y administración de riesgos con base en la acción decidida sobre una multiplicidad de factores intervinientes, pero entre los que destacan se encuentran el de peligro o amenaza, vulnerabilidad y capacidad.

La importancia que tiene la vida laboral en la vida general de cada persona es enorme, y los riesgos asociados al trabajo, durante mucho tiempo, se focalizaron en aspectos físicos, químicos y ergonómicos, sin tener herramientas de análisis de los Riesgos Psicosociales Laborales, de una gran importancia en el día de hoy. Cabe destacar la decidida acción de los organismos internacionales y los gobiernos que han transformado esta inicial preocupación en protocolos de riesgos de salud para los trabajadores. Todos estos esfuerzos decantan positivamente en la salud y calidad de vida de los trabajadores y sus seres cercanos.

Las organizaciones en este siglo han advertido que parte importante de la sustentabilidad y viabilidad de su actuar en los negocios pasa por la salud mental de sus trabajadores, la que se erige como un recurso estratégico que brinda robustez organizacional y permite una adaptación a los distintos desafíos de la industria.

Es un deseo que la instalación de una cultura generativa de seguridad se instale en una comunidad. No sólo la forma de actuar de los trabajadores incorpore en todo momento la seguridad y la disciplina operacional, sino 
que también atraviese a los dominios comunitarios, familiares e individuales, en donde una real evaluación de los peligros, las vulnerabilidades y las capacidades de respuesta, permitan reducir al máximo los riesgos y, en consecuencia, los efectos perjudiciales para las personas.

\section{Referencias}

Domjan, M. (2010). Principios de aprendizaje y conducta, 6ta Edición, México D.F: Wadsworth Cengage Learning Editores.

Instituto de Seguridad Laboral (2017). Plan Nacional de Seguridad y Salud en el Trabajo. Gobierno de Chile. Recuperado de http://www. isl.gob.cl/wp-content/uploads/2017/05/Plan-de-Salud-y-Seguridad-en-el-Trabajo-Instituto-de-Seguridad-Laboral-2017.pdf

Kamlot, D. (2017). Resiliência organizacional e marketing social: uma avaliação de fundamentos e afinidades. Cadernos EBAPE.BR, 15(spe), 482-495. https://dx.doi.org/10.1590/1679-395160269

Organización Mundial de la Salud [OMS] (1997). Estado del arte en resiliencia. Washington: Organización Panamericana de la Salud, Oficina Sanitaria Panamericana, Oficina Regional de la Organización Mundial de la Salud. Recuperado de http://wwwl.paho.org/hq/dmdocuments/2009/Resil6x9.pdf

Ministerio de Protección Social (2007). Herramientas para promover la Estrategia de la Seguridad del Paciente en el Sistema Obligatorio de Garantía de Calidad de la Atención en Salud. Bogotá: Editorial Fundación FITEC. Recuperado de https://www.minsalud.gov.co/ sites/rid/1/Herramientas\%20para \%20la\%20Seguridad\%20del\%20 Paciente.pdf

Ministerio de Salud (2017). Actualización del Protocolo de Vigilancia de Riesgo Sicosocial Laboral, Núm. 1.433 exenta. Santiago de Chile, 10 de noviembre de 2017. Recuperado de http://www.ist.cl/actualizacion-protocolo-riesgos-psicosociales-niveles-de-riesgo-dimensiones-resolucion-1433/

Organización Internacional del Trabajo [OIT] (1984) Factores psicosociales en el trabajo. Naturaleza, incidencia y prevención. Informe del Comité凶 Mixto OIT-OMS sobre Medicina del Trabajo, novena reunión, Ginebra, 18-24 de septiembre de 1984. Serie Seguridad, Higiene 
y Medicina del Trabajo $N^{\circ}$ 56. Ginebra: Oficina Internacional del Trabajo.

Parker, D., Lawrie, M., \& Hudson, P. (2006). A framework for understanding the development of organisational safety culture. Safety Science 44(6), 551-562. https://doi.org/10.1016/j.ssci.2005.10.004

Reason, J., Manstead, A., Stradling, S., Baxter, J. \& Campbe1l, K. (1990). Errors and violations on the roads: a real distinction?. Ergonomics, 33(10/11), 1315-1332. https://doi. org/10.1080/00140139008925335

Rutter, M. (2012). Resilience as a dynamic concept. Development and Psychopathology, 24(2), 335-344. https://doi.org/10.1017/ S0954579412000028

Salanova, M, Llorens, S. \& Martínez, I. (2016). Aportaciones desde la Psicología Organizacional Positiva para desarrollar organizaciones saludables y resilientes. Papeles del Psicólogo, 37(3), 177-184. Recuperado de http://www.papelesdelpsicologo.es/pdf/2773.pdf

Superintendencia de Seguridad Social (2016). Manual del Método del Cuestionario SUSESO/ISTAS 21 Versiones completa y breve, Gobierno de Chile. Recuperado de http://www.uchile.cl/ documentos/manual-del-metodo-del-cuestionario-susesoistas21-2016_119348_0_3619.pdf

Ulloa, F. (2011). Manual de gestión de riesgos de desastre para comunicadores sociales: una guía práctica para el comunicador social comprometido en informar y formar para salvar vidas. Lima: UNESCO Office Lima. Recuperado de https://unesdoc.unesco.org/ark:/48223/ pf0000219184

Xenidis, Y. \& Theocharous, K. (2014). Organizational health: Definition and assessment. Procedia Engineering, 85, 562 - 570. https://doi. org/10.1016/j.proeng.2014.10.584 